\title{
HOMOTOPY EQUIVALENCE OF FIBER BUNDLES
}

\author{
M. L. CURTIS AND R. LASHOF
}

1. Introduction. A classification of bundles has been given by Lashof [3] by mapping the loop space of the base into the structure group. This is extended here to a classification of bundles under fiber homotopy equivalence. The result does not agree with Theorem 10.1 of [1], and that theorem can be seen to be false by simple examples. (The error responsible occurs in the next-to-last sentence of the proof of Lemma 8.3.)

Since tangent sphere bundles given by different differentiable structures on a manifold are fiber homotopy equivalent (see [9] and [6]) our classification theorem yields some characteristic classes which are independent of the differentiable structure. These classes are in the cohomology ring of the loop space of the manifold instead of the manifold itself. ${ }^{1}$ The classification theorem is given in $\$ 2$ and application to sphere bundles is made in $\S 3$.

2. The classification theorem. We use the notation and results of [3]. We require that the base space $B$ be a polyhedron because this allows us to use a result of $A$. Dold [2] to simplify both the statement and proof of the theorem. We will assume that the fiber $F$ is locally compact and let $M$ be the space of maps $F \rightarrow F$ which have homotopy inverses, topologized with the compact-open topology. Finally, we assume that the structure group $G$ has the compact-open topology so that there is an inclusion map $j: G \rightarrow M$. We recall that for a bundle $(E, B, F)$ one obtains a homomorphism $\phi$ from the groupoid $\Omega$ of loops in $B$ into the structure group $G .{ }^{2}$ Let $P$ be the space of paths from a point $b_{0}$ of $B$.

Presented to the Society, September 1, 1955; received by the editors August 30, 1957.

1 These classes are just the suspension in the space of paths of the bundle characteristic classes. For, if $\left(E_{G}, B_{G}, G\right)$ is the universal bundle for the group $G$, the map $f: B \rightarrow B_{G}$ which induces a given bundle $(E, B, G)$ with group $G$, defines a fiber preserving map $f^{\#}:(P, B, \Omega) \rightarrow\left(E_{G}, B_{G}, G\right)$ by lifting the map $P \rightarrow B \rightarrow B_{G}$ into $P \rightarrow E_{G}$. It is easy to see that the map $f^{y}: \Omega \rightarrow G$ is homotopic to the groupoid homomorphism $\phi: \Omega \rightarrow G$ of the given bundle. Since suspension on cohomology commutes with the fiber preserving map $\left(f^{\sharp}\right)^{*}$, and since the cohomology of $G$ is generated by the suspension of the generators of the cohomology ring of $B_{G}$ (as proved by A. Borel), $\phi^{*}\left(H^{*}(G)\right.$ ) $=\left(f^{\#}\right)^{*}\left(H^{*}(G)\right)$ is just the suspension of the characteristic subring of $H^{*}(B)$.

${ }^{2}$ Since we are taking $B$ to be a polyhedron, it follows from Milnor's construction [5] that $\Omega$ may be replaced by a group and $\phi$ by a homomorphism of that group into $G$. One then gets a theorem analogous to Theorem 2.1 with Milnor's space $\widetilde{E}$ $[5$, p. 274] replacing the path space $P$. 
Theorem 2.1. Two bundles $\left(E_{1}, B, F\right)$ and $\left(E_{2}, B, F\right)$ with structure group $G$ are fiber homotopy equivalent if and only if there exists a map $\psi: P \rightarrow M$ such that

$$
j \phi_{2}(p, q) \psi(q)=\psi(p) j \phi_{1}(p, q) .
$$

Proof. We recall from [3, p. 442] that $\phi_{1}$ and $\phi_{2}$ arise from bundle maps $R_{i}: P \times F \rightarrow E_{i}, i=1,2$, by the definition $\phi_{i}(p, q)=p_{i}^{*-1} q_{i}^{*}$ where $p_{i}^{*}$ denotes the map $R_{i} \mid p \times F$.

Given a fiber homotopy equivalence $f: E_{1} \rightarrow E_{2}$, we define $\psi(p)$ $=p_{2}^{*-1} p_{1}{ }^{*}$. Let $(p, q) \in \Omega$ and we calculate

$$
\begin{aligned}
\psi(q) & =q_{2}^{*-1} f q_{1}^{*}=q_{2}^{*-1} p_{2}^{*} p_{2}^{*-1} f p_{1}^{*} p_{1}^{*-1} q_{1}^{*} \\
& =\phi_{2}(q, p) \psi(p) \phi_{1}(p, q) .
\end{aligned}
$$

Since $\phi_{2}(q, p)$ is the inverse of $\phi_{2}(p, q), \psi$ has the required property.

Conversely, suppose we are given such a map $\psi$. For each fiber $F_{b}$ of $E_{1}$ we choose a path $p$ from $b_{0}$ to $b$ and define $f \mid F_{b}=p_{2}^{*} \psi(p) p_{1}^{*-1}$. Then $f$ is well defined, for if we had chosen a path $q$ instead of $p$ we would have

$$
\begin{aligned}
& f \mid F_{b}=\stackrel{*}{q_{2}} \psi(q) q_{1}^{*-1}=\stackrel{*}{q_{2} \phi_{2}}(q, p) \psi(p) \phi_{1}(p, q) q_{1}^{*-1} \\
& =q_{2}^{*} q_{2}^{*-1} p_{2}^{*} \psi(p) p_{1}^{*-1} q_{1}^{*} q_{1}^{*-1}=p_{2}^{*} \psi(p) p_{1}^{*-1} .
\end{aligned}
$$

Since $\psi(p) \in M, f$ is a homotopy equivalence on each fiber. It follows from [2, p. 120] that $f$ is a homotopy equivalence, and the theorem is proved.

Let $H^{*}(A)$ denote the integral cohomology ring of a space $A$.

Corollary. If $E_{1}$ and $E_{2}$ are fiber homotopy equivalent, then $\left(j \phi_{1}\right)^{*}\left(H^{*}(M)\right)$ and $\left(j \phi_{2}\right)^{*}\left(H^{*}(M)\right)$ are isomorphic subrings of $H^{*}(\Omega)$.

Proof. By Theorem 2.1 there exists a map $\psi: P \rightarrow M$ such that $j \phi_{2}(p, q) \psi(q)=\psi(p) j \phi_{1}(p, q)$. We shall show that this implies our result. Let $\Gamma: P \times I \rightarrow P$ be the standard contraction of $P$ to the null path $n_{0}: I \rightarrow b_{0}$. Define a homotopy $\phi: \Omega \times I \rightarrow M$ by

$$
\phi(p, q, t)= \begin{cases}\psi(\Gamma(p, 1-2 t)) j \phi_{1}(p, q) & \text { for } 0 \leqq t \leqq 1 / 2, \\ j \phi_{2}(p, q) \psi(\Gamma(q, 2 t-1)) & \text { for } 1 / 2 \leqq t \leqq 1\end{cases}
$$

Then $\phi$ is well defined since $\phi(p, q, 1 / 2)=\psi(p) j \phi_{1}(p, q)=j \phi_{2}(p, q) \psi(q)$. Also we see that 


$$
\begin{aligned}
& \phi(p, q, 0)=\psi\left(n_{0}\right) j \phi_{1}(p, q), \\
& \phi(p, q, 1)=j \phi_{2}(p, q) \psi\left(n_{0}\right)
\end{aligned}
$$

so that $\psi\left(n_{0}\right) j \phi_{1}$ is homotopic to $j \phi_{2} \psi\left(n_{0}\right)$. This proves the corollary, since $\psi\left(n_{0}\right)$ has a homotopy inverse.

REMARK. If $f$ is homotopic to the identity on the fiber over $b_{0}$ then $\psi\left(n_{0}\right)$ is the identity and $\left(j \phi_{1}\right)^{*}=\left(j \phi_{2}\right)^{*}$. In particular, if the fiber is a sphere, then the orientations of the fibers of the two bundles may be so chosen that this is the case.

3. Application to sphere bundles. Thom [9] and Nash [6] have shown that any two differentiable structures on an $n+1$ manifold give homotopy equivalent tangent sphere bundles. In view of the corollary in the preceding section, the corresponding maps $j \phi_{1}$ and $j \phi_{2}$ give isomorphic subrings $\left(j \phi_{i}\right) *\left(H^{*}(M)\right)$ of the characteristic subrings $\phi_{i}^{*}\left(H^{*}\left(O_{n+1}\right)\right)$, where $O_{n+1}$ is the orthogonal group. Hence we are led to study the cohomology homomorphism induced by the inclusion $O_{n+1} \rightarrow M$. Both $O_{n+1}$ and $M$ consist of two equivalent components so we may as well study the inclusion $i: R_{n+1} \rightarrow H$, where $R_{n+1}$ is the rotation group of $S_{n}$ and $H$ consists of all maps $S_{n} \rightarrow S_{n}$ of degree +1 .

If we choose a point $y_{0} \in S_{n}$, then the map $\pi: H \rightarrow S_{n}$ defined by $\pi(h)=h\left(y_{0}\right)$ is a fibering (see $[10$, p. 461]). We have

$$
\Omega \stackrel{\phi}{\rightarrow} R_{n+1} \stackrel{i}{\rightarrow} H \stackrel{\pi}{\rightarrow} S_{n}
$$

and, since $(i \phi)^{*}\left(H^{*}(H)\right)$ is unique (up to isomorphism) for homotopy equivalent bundles, so is $(\pi i \phi)^{*}\left(H^{*}\left(S_{n}\right)\right)$. Information about the homomorphism $(\pi i)^{*}: H^{n}\left(S_{n}\right) \rightarrow H^{n}\left(R_{n+1}\right)$ is at hand (see $[8$, p. 120] and $[4])$, and combines with the results above to give the following information.

Theorem 3.1. The image $(\pi i)^{*}\left(H^{n}\left(S_{n}\right)\right) \subset H^{n}\left(R_{n+1}\right)$ is a homotopy invariant. If $n$ is odd, it is infinite cyclic and if $n$ is even it is cyclic of order 2.

CoRollary 3.1. If the base space is $S_{n+1}$ with $n$ odd, the even elements of $H^{n}(\Omega)$ are homotopy invariants.

In the remainder of this section we will obtain information about the map $\pi: H \rightarrow S_{n}$, with the idea that it combines with information about $\pi i$ to give information about $i$.

Theorem 3.2. If $n$ is odd, then $H_{p}(H, Z)$ is finite for all $p>0$ except $p=n$. 
Proof. Let $Q=\pi^{-1}\left(y_{0}\right)$. Since $Q$ is an iterated loop space, starting with $S_{n}$, the homotopy groups of $Q$ are known to be

$$
\pi_{0}(Q)=0, \quad \pi_{i}(Q)=\pi_{n+i}\left(S_{n}\right) \quad \text { for } i>0 .
$$

Serre [7] has shown that all of these groups are finite. Hence if $k$ is a field of characteristic 0 , then

$$
\pi_{i}(Q) \otimes k=0
$$

for all $i$.

Then $H_{p}(Q, k)=0$ for all $p$, so the Vietoris-Begle theorem implies that $H_{p}(H, k) \approx H_{p}\left(S_{n}, k\right)$, and this implies that $H_{p}(H, k)=0$ for all positive $p \neq n$. Since the groups are all finitely generated, it follows that the groups $H_{p}(H, Z)$ are finite except for $p=n$.

The fiber space $\left(H, S_{n}, \pi\right)$ has the interesting property that its homotopy sequence and its Wang sequence are formally the same. Actually, we will see that they are isomorphic modulo a field of characteristic 0 , for even $n$.

We start with the ladder for the pair $(H, Q)$.

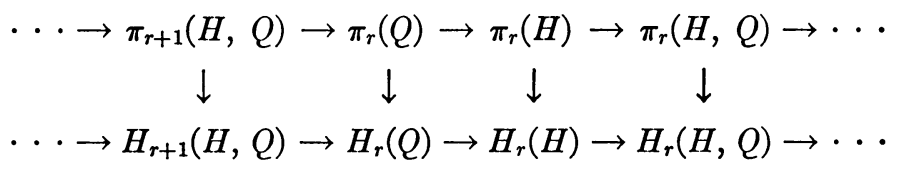

We use the Wang isomorphism $w: H_{r+n}(H, Q) \rightarrow H_{r}(Q)$ to obtain the Wang sequence from the homology sequence and use the projection isomorphism $p: \pi_{r+1}(H, Q) \rightarrow \pi_{r+1}\left(S_{n}\right)$ followed by the Hurewicz isomorphism $h: \pi_{n+r}\left(S_{n}\right) \rightarrow \pi_{r}(Q)$ in the homotopy sequence. This gives the ladder

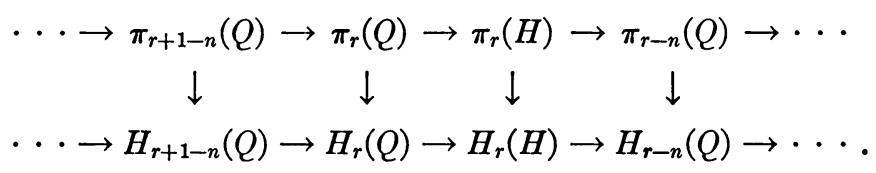

THEOREM 3.3. If $n$ is even, the mapping from the homotopy sequence to the homology sequence above is an isomorphism modulo a field $k$ of characteristic 0 .

PRoof. Since $n$ is even $\pi_{n-1}$ has a free cyclic subgroup. If $f: S_{n-1} \rightarrow Q$ represents a generator of this subgroup then $f$ induces isomorphisms

$$
\pi_{i}\left(S_{n-1}\right) \otimes k \rightarrow \pi_{i}(Q) \otimes k
$$

because $\pi_{i}(Q)$ is finite except for $i=n-1$. Since $H_{i}\left(S_{n-1}, k\right)$ is isomorphic with $\pi_{i}\left(S_{n-1}\right) \otimes k$, the same is true of $Q$ and the theorem follows. 
Using the above theorem and the suspension theorems, we get the following results.

For $n$ odd:

1. $H_{n}(H) \rightarrow H_{n}\left(S_{n}\right)$ is onto,

2. $H_{n-1}(Q) \approx H_{n-1}(H)$ and both are finite,

3. For $n>3, H_{1}(Q) \rightarrow H_{n}(Q)$ is either trivial or injective.

For $n$ even:

1. $H_{n}(H) \rightarrow H_{n}\left(S_{n}\right)$ is trivial, so

$$
H_{n}(H) \approx H_{n}(Q) \text { or } H_{n}(H) \approx H_{n}(Q) / Z_{2} .
$$

2. $I_{n-1}(I I) \approx H_{n-1}(Q) / Z$.

\section{BiBLIOGRAPHY}

1. M. L. Curtis, Classification spaces for a class of fiber spaces, Ann. of Math. vol. 60 (1954) pp. 304-316.

2. A. Dold, Über faserweise Homotopieäquivalenz von Faserräumen, Math. Zeit. 62 (1955) pp. 111-136.

3. R. Lashof, Classification of fiber bundles by the loop space of the base, Ann. of Math. vol. 64 (1956) pp. 436-446.

4. C. E. Miller, The topology of rotation groups, Ann. of Math. vol. 57 (1953) pp. 90-114.

5. John Milnor, Construction of universal bundles, I., Ann. of Math. vol. 63 (1956) pp. $272-284$.

6. J. Nash, A path space and the Sticfel-Whitney classes, Proc. Nat. Acad. Sci. U.S.A. vol. 41 (1955) pp. 320-321.

7. J. P. Serre, Homologie singulière des espaces fibrés. Applications, Ann. of Math. vol. 54 (1951) pp. 425-505.

8. N. Steenrod, Topology of fibre bundles, Princeton, 1951.

9. R. Thom, Espaces fibrés en sphères et carrés de Stecnrod, Ann. École Norm. (1952) pp. 109-182.

10. G. Whitehead, On products in homotopy groups, Ann. of Math. vol. 47 (1946) pp. $460-475$.

UNIVERSITY OF GEORGIA AND

University of Chicago 